\title{
Wave and turbulence measurements at a tidal energy site
}

\author{
Jean-François Filipot \\ France Energies Marines \\ 15 rue Johannes Keppler \\ Brest, France \\ Email: jean.francois.filipot \\ @ france-energies-marines.org prevosto@ifremer.fr \\ ZI Pointe du diable
}

\author{
Marc Prevosto \\ Ifremer, \\ 29280 Plouzané \\ Email: marc. \\ Christophe Maisondieu \\ Ifremer, \\ ZI Pointe du diable \\ 29280 Plouzané \\ Email: christophe. \\ maisondieu@ifremer.fr
}

\author{
Marc Le Boulluec \\ Ifremer, \\ ZI Pointe du diable \\ 29280 Plouzané \\ Email: marc.le.boulluec \\ @ifremer.fr
}

\author{
Jim Thomson \\ U. of Washington, \\ Seattle \\ WA 98195-2600 USA \\ Email: jthomson@ \\ apl.washington.edu
}

\begin{abstract}
This work presents the analysis of wave and turbulence measurements collected at a tidal energy site. A new method is introduced to produce more consistent and rigorous estimations of the velocity fluctuations power spectral densities. An analytical function is further proposed to fit the observed spectra and could be input to the numerical models predicting power production and structural loading on tidal turbines. Another new approach is developed to correct for the effect of the Doppler noise on the high frequencies power spectral densities. The analysis of velocity time series combining wave and turbulent contributions demonstrates that the turbulent motions are coherent throughout the water column, rendering the wave coherence-based methods not applicable to our dataset. To avoid this problem, an alternative approach relying on the pressure data collected by the ADCP is introduced and shows appreciable improvement in the wave-turbulence separation.
\end{abstract}

\section{INTRODUCTION}

Tidal turbines are meant to be deployed in highly energetic locations where tidal currents are strong and flow highly variable. Turbine design requires a precise knowledge of such high velocity flow, not just for production optimization but also for an accurate characterization and description of the loadings inducing structural fatigue on the devices. Inflow conditions are of prime importance to numerical ([1]) and physical ([2]) modeling. Various elements generate flow turbulence in this energetic environment. The flow being considered constrained between two walls (the sea-bed and the free surface), different phenomena occur and are responsible for its variability. Viscosity causes shear and turbulence in the bottom boundary layer that can be advected by the mean flow. Near the surface, atmospheric constraints may induce additional turbulence, usually limited to the upper boundary layer. In the presence of wave breaking, an important momentum flux is generated downwards with a high dissipation rate ([3]) and is sometimes associated with coherent eddies propagating downstream. In addition to these turbulent features, the flow is generally altered by the irrotational wave kinematics, throughout the water column [4].

Over recent years, large efforts have been made toward the characterization of these complex flows through analysis of measurements of Acoustic Doppler Velocimeters (ADVs) and Acoustic Doppler Current Profilers (ADCPs) ([5], [6], [7]). Even though ADVs allow high frequency measurement of the local velocity, hence providing access to local turbulence
([8]), ADCPs are often preferred as they allow current profile measurements along the water column with one single device. However, using ADCPs has some major drawbacks, especially when investigating flow variability and turbulence. One of which is the presence of higher Doppler noise, requiring a proper filtering. The validity of the assumptions made for deriving true velocities in the geographic referential from the along beam velocities [9] is also questionable as is Taylor's frozen field assumption for turbulent scales estimation. Nevertheless, methods have been developed and presented by various authors, especially regarding the separation of the wave and turbulence spectra. [10] presented a method based on the structure function method for the assessment of the rate of Turbulent Kinetic Energy dissipation. The variance fit method for removing the wave component in the spectra, proposed by [11] was further discussed in [12] indicating the method could be efficient provided the phase coherence assumption stands. The method was also used with good confidence by [7] for an analysis of variable wind and waves on tidal flow in the eastern English Channel. [4] presented a linear filtration method, requiring assessment of the coherence between the local and depth-averaged velocity components.

In the present paper, we attempt to provide a valuable contribution to the characterization of the flow variability and turbulence by means of ADCP measurements analysis, discuss existing methods and propose alternative solutions to reduce the noise and accurately distinguish the wave and turbulence contribution to the velocity spectrum.

In this paper, we shall explore the turbulent features of the flow, based on a signal sample with negligible wave energy. The proposed method allows to correct for the non-stationary nature of the turbulent flow and provides reliable and consistent turbulence spectra. In a second section, we attempt to extract the wave and turbulence spectral signatures. As the coherence method appeared to fail at our site, an alternative approach, using the pressure collected by the ADCP is proposed.

\section{DATA SET}

\section{A. Site description}

Data used for this analysis was extracted from a dataset recorded during a survey conducted in 2013 at the PaimpolBréhat pilot tidal energy farm managed by Electricité de France. The considered site is located in the north-east of 
Bréhat Island in the western English Channel, close to the coast of Brittany, France (Figure 1). The location is known for

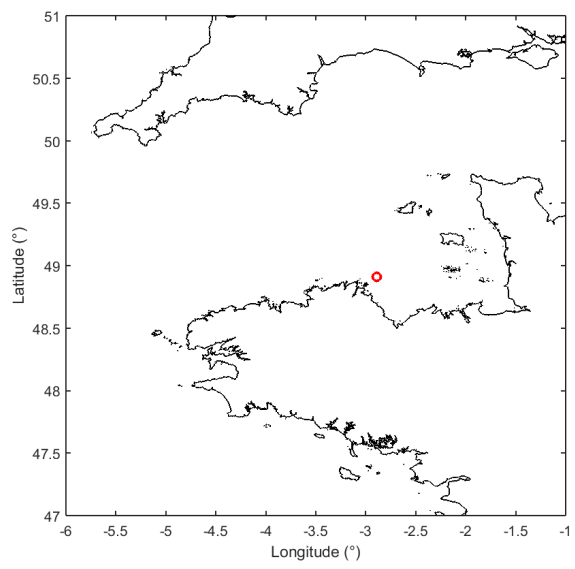

Fig. 1. Measurement site location in the English Channel (red circle).

its strong tidal currents, with velocities up to $3 \mathrm{~m} / \mathrm{s}$ and has been identified as a potential site for the deployment of tidal turbines, whether for the testing of prototypes or for electricity production with a connection to the grid. Even though lying in a relatively sheltered coastal area, the site is submitted to a complex wave climate, dominated by long northwesterly swells and shorter northeasterly wind seas.

Wave information was available from the ADCP deployed at the site, characterization of the wave climatology was completed by an analysis of 11-year time series (2002-2012) of wave parameters. This was obtained from a high resolution hindcast database ([13]), built running the code WaveWatch III $^{\circledR}$ on an unstructured grid and accounting for tidal currents and water levels.

Sea-states are usually moderate with an average significant wave height of $1.2 \mathrm{~m}$ and a maximum identified at $6.3 \mathrm{~m}$. Peak periods are of about $10 \mathrm{~s}$ on average but longer swells with peak periods above $20 \mathrm{~s}$ were also identified. Most dominant wave trains propagate from the northwest direction $\left[280^{\circ}-320^{\circ}\right]$ but wind-sea events, with shorter periods, are also identified in the north to east sector. Scatter plot on Figure 2 presenting wave peak period as a function of peak direction and significant wave height summarizes this global wave climatology.

As we are mainly interested here in the influence of waves on flow variability and the alteration it can induce on current and turbulence measurement with ADCPs, it is interesting to investigate the horizontal component of the wave orbital velocity in the water column. Values of this horizontal velocity at turbine hub elevation were extrapolated from the RMS values near the sea-bed that were directly evaluated from the wave spectra, according to equation 1 hereafter [14]:

$$
U_{w, r m s}=\sqrt{\int_{0}^{2 \pi} \int_{0}^{\infty} \frac{\sigma^{2}}{\sinh ^{2}(k d)} S(k, \theta) d k d \theta}
$$

where $S$ is the wave spectrum, $\sigma$ is the angular-frequency, $k$ the wavenumber, $\theta$ the direction and $d$ the water depth. Average RMS horizontal orbital velocity is found to be of

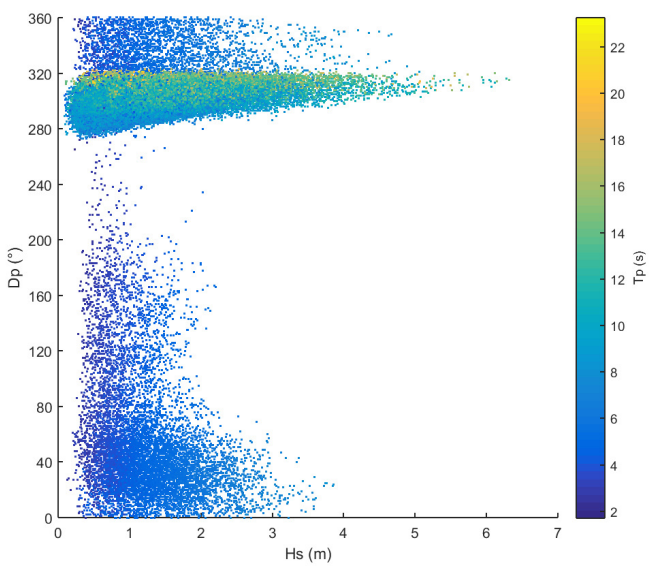

Fig. 2. Wave peak period as a function of peak direction and significant wave height at the field experiment site, from the 11-year model dataset.

about $0.17 \mathrm{~m} / \mathrm{s}$ at tidal turbine hub elevation, maximum value is found to be of about $1.4 \mathrm{~m} / \mathrm{s}$, so that wave kinematics at this location should be taken into account when assessing flow variability and turbulence.

\section{B. Current Dataset}

Data used for this work was extracted from a dataset recorded during a two months survey conducted in 2013 at the Paimpol-Bréhat site where a 4-beam TRDI Workhorse sentinel $600 \mathrm{kHz}$ ADCP had been moored to the sea-bed in $43 \mathrm{~m}$ water depth. Tidal range at the site can approach up to about $10 \mathrm{~m}$ during spring tides. Data was quality controlled ([15]) and bins 3 to 15 ranging from 6.04 meters above the bottom (m.a.b) to 26.44 m.a.b were conserved for analysis as $100 \%$ of the velocity data passed the quality control. Bin size was $1.71 \mathrm{~m}$. The ADCP was slightly tilted $\left(-1.5^{\circ}\right.$ in pitch, $-0.5^{\circ}$ in roll). Standard deviation of pitch, roll and yaw remained below $1^{\circ}$ over the whole record duration, within the range recommended by [9]. Horizontal $\mathrm{u}, \mathrm{v}$ and vertical $\mathrm{w}$, components of the current velocity were recomposed in the geographic referential from the four beam velocities using the classical method by [9]. $20 \mathrm{mn}$-averaged velocities were derived from the instantaneous velocities (sampling frequency $1 \mathrm{~Hz}$ ). An asymmetry of the flow was observed between ebb and flood in both intensity and direction. Velocities are higher during flood, having reached a maximum of $3.0 \mathrm{~m} / \mathrm{s}$ at 28.8 m.a.b. while during ebb, the maximum was only measured at $2.1 \mathrm{~m} / \mathrm{s}$. Flood direction is oriented towards the East $\left(100^{\circ}\right)$ while the ebb direction is towards north-west $\left(300^{\circ}\right)$. Such a misalignment of about $20^{\circ}$ is most likely due to the influence of the local bathymetry. As a consequence, the flow is generally facing long swells during ebb (see Figure 2), while it will be following them at an angle of about $20^{\circ}$ to $40^{\circ}$ during flood. Data used in the present work come from three continuous 24-hour subsets of this record (April 7, 10, 14). They were specifically chosen for the purpose of this study on the influence of waves on the measurements of the flow variability and turbulence by means of an ADCP. Hence they consist in two 24-hour records (April 10 and 14) with long period swells (peak period of 14 to $15 \mathrm{~s}$ ) with substantial 


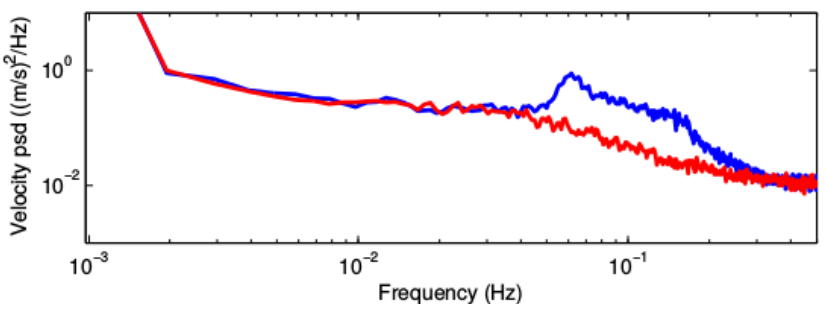

Fig. 3. Spectral density of current velocities measurements. In red without waves (April 7), in blue with waves (April 10).

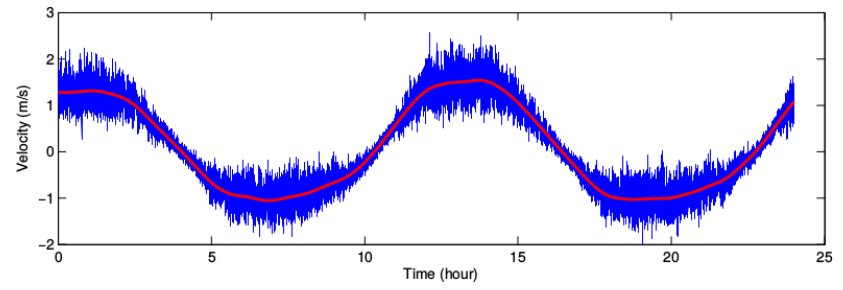

Fig. 4. 24h time series of east-west current velocities (blue curve). Low frequency tidal part (red curve).

energy (significant wave height of 1.5 to $3 \mathrm{~m}$ ) and one 24$\mathrm{h}$ record (April 7) with short, low energy wave field. All three records were chosen during spring tide periods with peak maximum tidal velocities of about $2.5 \mathrm{~m} / \mathrm{s}$.

\section{TURBULENCE SPECTRA IN THE ABSENCE OF WAVES}

\section{A. Preliminary observations of velocity spectra}

Velocity spectra estimated from a direct Fourier analysis of the velocity time series extracted from the dataset all show the same classical features. As clearly visible on Figure 3, they can be split into three different parts. The lower frequency range, for frequencies below $0.035 \mathrm{~Hz}$, is related to the general tidal circulation. In the range of higher frequencies up to $0.3 \mathrm{~Hz}$, including the inertial range, the velocity spectrum is regularly decreasing and can be largely affected by waves in situations when the significant wave height is high (see Figure 3). Finally the upper part of the frequency band, where the spectrum flattens, above $0.3 \mathrm{~Hz}$, is generally considered as dominated by the Doppler noise ([16], [8]).

We shall first analyze the turbulence when the effect of waves is not detectable. The examination of combined wave and turbulent signals will be presented in section IV. Figure 4 gives an example of the east-west component of the velocity measured during 24 hours. The tidal-induced modulation of high frequency turbulence is evident in this illustration. The extraction of the tidal part (red curve) is obtained by using a cubic smoothing spline. To derive more general results from the east-west/north-south measurements, a rotation is applied to transform the time series in a frame defined by the main direction of the tidal current. In the following we will call principal current, the current in the tidal direction and secondary current, the current in the orthogonal direction.

\section{B. Doppler Noise Characteristics}

Indeed, a large number of methods presented in the literature for the detection and filtering of the Doppler noise are

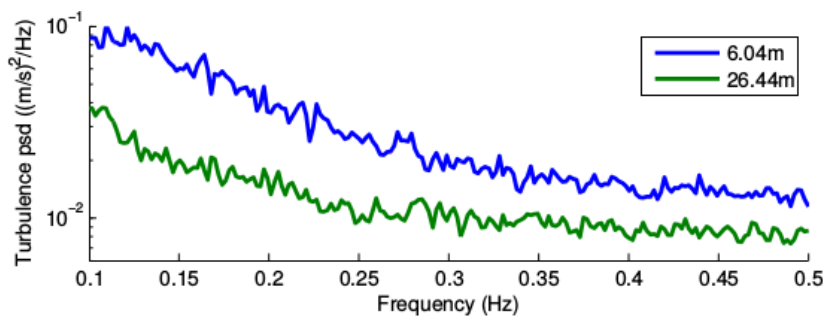

Fig. 5. Frequency tails of the turbulence measurements, in the principal direction.

based on the assumption that this noise can be assimilated to a white noise whose variance amplitude equals the value of the plateau in the tail of the spectrum. These methods were applied to the case of low energy flows with lower high frequency turbulence and for velocity time series measured by mean of ADVs allowing a sampling frequency about ten times higher than the typical sampling frequency of an ADCP ([8], [17], [18], [19]). The noise variance is estimated in the tail of the spectrum, that is at frequencies close to the Nyquist frequency which, for an ADCP would be relatively low (i.e. $0.5 \mathrm{~Hz}$ for a $1 \mathrm{~Hz}$ sampling frequency) compared to the typical turbulence frequencies but also to wave components. Hence, the validity of the assumption of the saturation of the variance by only Doppler noise is questionable in the case of an ADCP and is worth investigation. In the following, and as said earlier, we consider three $24 \mathrm{~h}$ time series of the velocities recorded with a $1 \mathrm{~Hz}$ sampling frequency for three different dates: April 7, 10 and 14. On April 7, the significant wave height was low and the peak period relatively short. This record is considered as a reference without waves while stronger wave conditions were observed for the two other dates. As already mentioned, the presence of the Doppler noise can be identified in the high frequency tail of the spectral density. A zoom on the tail of the spectra calculated for the lower and upper bins of the ADCP (elevation 6.04 m.a.b and 26.44 m.a.b, respectively) is presented in figure 5 for the date of April 7 and shows the saturation of the spectral densities, marked by a flattening, when the noise becomes dominant.

In order to estimate the variance of the noise, we first consider its value on the frequency band $[0.44 \mathrm{~Hz}-0.5 \mathrm{~Hz}]$. We then make the assumption that the Doppler noise is a white noise leading to a direct estimate its total variance over the whole frequency range $[0 \mathrm{~Hz}-0.5 \mathrm{~Hz}]$. The variance of the noise estimated on all 13 bins between $6.04 \mathrm{~m}$ and $26.44 \mathrm{~m}$, shows (Figure 6) a linear relation with the velocity variance estimated on April 7 (the highest turbulence corresponds to the deepest bin). The same procedure was applied to the two other datasets. A same linear relation between turbulence and noise could be identified but with a higher slope coefficient (Figure 7). The part of the velocity variance due to waves (present in series 04/14 and 04/10) seems not to participate in the same way in the Doppler noise. Applying the same procedure again for the three time series but assessing the variance of the velocity corresponding to the lower frequency range only, [0 $\mathrm{Hz}-0.035 \mathrm{~Hz}$ ], that is for components with frequencies below the wave frequency range, the linear trend between noise and velocity variance is found to be about the same (Figure 8). This result seems to indicate that Doppler noise is not generated in the wave frequency band with $f>0.035 \mathrm{~Hz}$. 


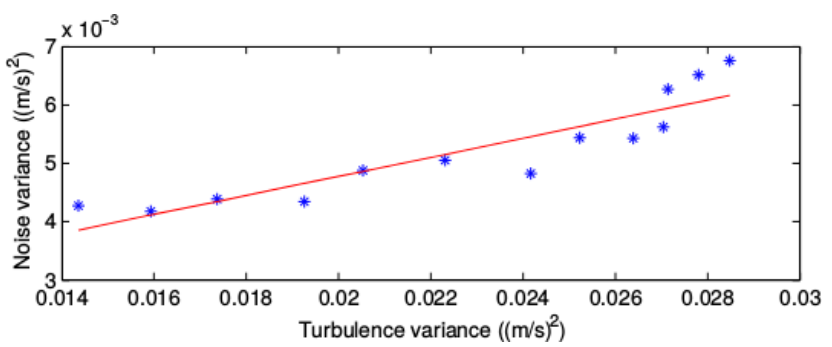

Fig. 6. Frequency tails of the turbulence measurements, in the principal direction.

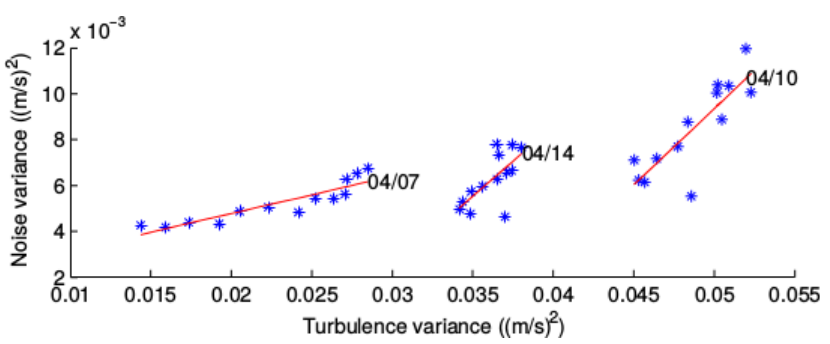

Fig. 7. Frequency tails of the turbulence measurements, in the principal direction.

\section{Estimation of a turbulence spectrum model}

When subtracting the tidal part of the current from the total current (which gives the turbulent part), the effect of the modulation of the turbulence by the tidal current is even clearer (Figure 9). It shows that the tidal current generates turbulence at practically the same intensity in the direction of the tidal current and in the orthogonal direction. Because this turbulence signal is clearly non steady, at least in terms of variance, a direct estimation of the spectral density from the turbulence time series would have no meaning. However, this second order non-stationarity can be corrected in estimating a relation between the instantaneous tidal current and the instantaneous variance of the turbulence. This relation can be derived by classifying the tidal current amplitude into bins of fixed width over the range of observed mean tidal velocities and evaluating the associated variance of the turbulence for each bin. An analytic function can then be fitted to this distribution. In the present case a function in the form :

$$
f(x)=a \cos (x+\phi)+b \cos (2 x+\psi)
$$

was found to provide a good agreement. An example of the result of the application of this procedure to the signal of the principal component of the turbulence at one ADCP bin level is

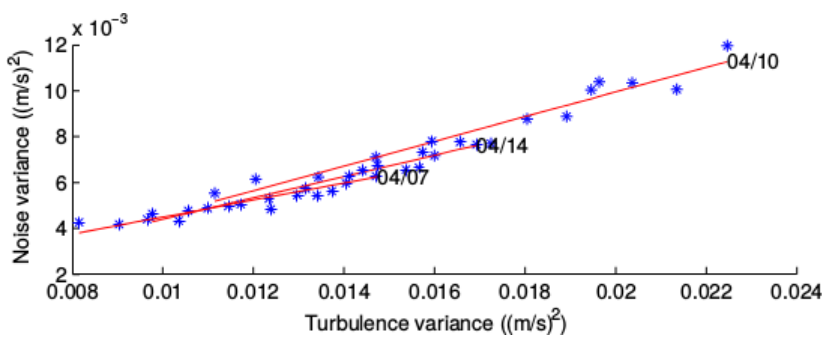

Fig. 8. Low frequency turbulence vs Noise variances for the three time series.

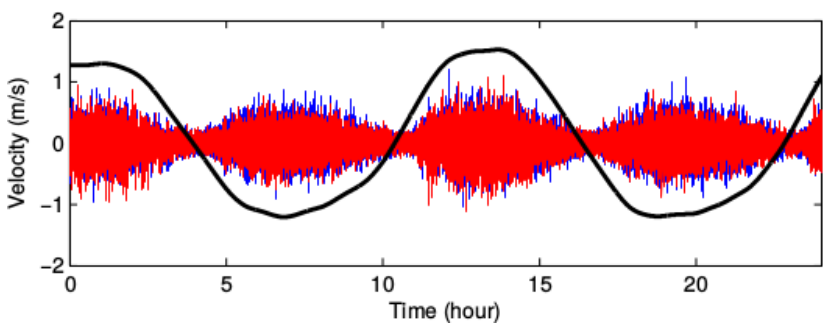

Fig. 9. Tidal and turbulence current velocities. Tidal current (black), principal turbulance (blue), orthogonal turbulence (red)

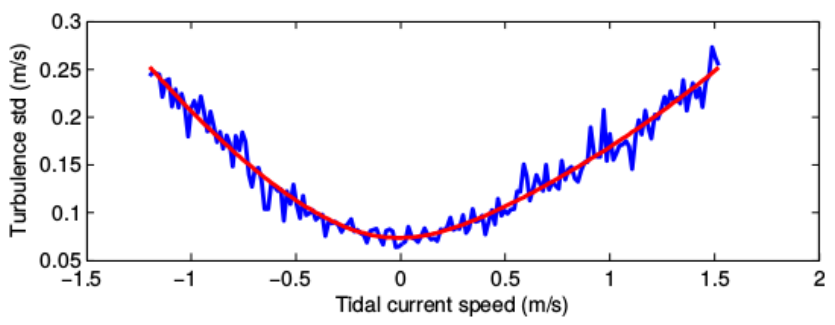

Fig. 10. Estimation and fitting of the correction function.

presented in Figure 10 where the turbulence standard deviation (instead of the variance) of the turbulence as a function of the the tidal velocity is plotted. This same procedure is applied to the signals of both principal and orthogonal velocities for the 13 levels of the ADCP and results for the principal component are presented Figure 11 (Level 1, resp. 5, 13 correspond to elevations 6.04 m.a.b, resp. 12.84 m.a.b, 26.44 m.a.b). This results again shows the decrease of the turbulence with the elevation in the water column, except for slack waters, with velocities in the range $[-0.3 \mathrm{~m} / \mathrm{s}, 0.3 \mathrm{~m} / \mathrm{s}]$. Moreover, a clear asymmetry is evident between ebb and flood, with higher turbulence standard deviation values observed with negative mean current velocities. A reason for this is probably due to the local bathymetry downstream and upstream. Similar results are found when considering the orthogonal components.

In a second step, these analytical correction functions are used to stationarize the time series. Figure 12 shows an example of resulting time series. Spectral densities can now been estimated on all the levels and for principal and secondary directions. A periodogram method is used with Hanning windowing $17 \mathrm{mn} 4 \mathrm{~s}$ long. Figure 13 suggests that if for the orthogonal direction the spectral densities are quite similar, in the principal direction some spectra seem to deviate from the others. The slopes appear milder than the classical $5 / 3$ one, but as we will see in section IV, this is mainly due to

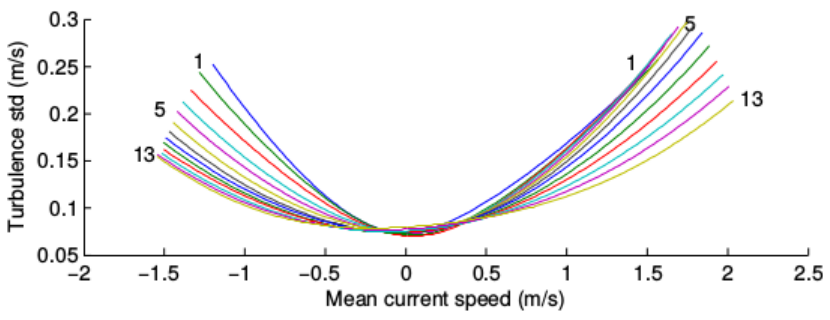

Fig. 11. Fitting of the correction function for the 13 levels in the principal direction. 


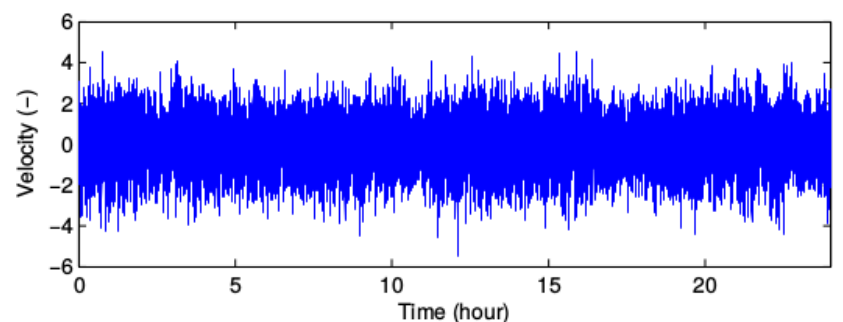

Fig. 12. Turbulence time series after stationarization.
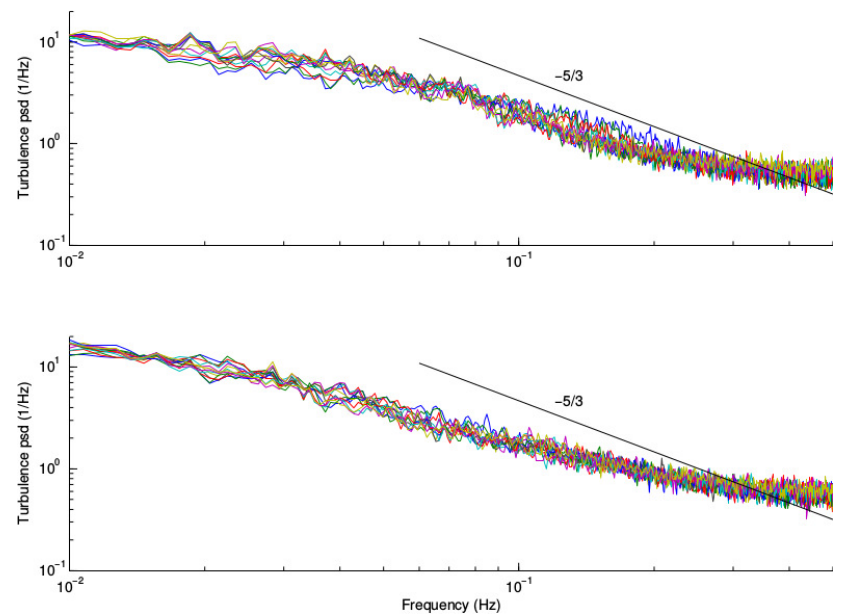

Fig. 13. Spectral densities of the turbulence time series after stationarization for the 13 levels. Primary direction (top), secondary direction (bottom).

the effect of the Doppler noise. The shape of these spectra basically consists in two constant zones, at low and high frequencies, connected by a negative slope in the intermediate range. Hence, it makes sense to build a model of these spectra based on an analytical function in the form:

$$
S(f)=\frac{1}{\alpha f^{n}+\beta}+\epsilon
$$

$1 / \beta+\epsilon$ corresponds to the value of the constant zone at low frequency whilst $\epsilon$ is the noise at high frequencies and - $n$ corresponds to the slope. $\alpha, \beta, \epsilon$ and $n$ are found in order to minimize the mean square error between the logarithms of the empirical and model spectra. The fitting is plotted Figure 14 without considering the $\epsilon$ term which models the Doppler noise. The slopes are quite close to the $-5 / 3$ value for the levels near the sea bottom (top extreme blue curve for the principal direction, bottom extreme curves for the secondary direction), but the slopes tend to increase (resp. decrease) in primary (resp. secondary direction) with the altitude above the bottom.

\section{SEPARATION OF WAVE AND TURBULENCE SPECTRA}

\section{A. Noise correction method}

ADCP measurements are contaminated with the so-called Doppler noise that arises when estimating a unique Doppler shift from finite length acoustics pulses (e.g. [8], [19]). To suppress the Doppler noise from our measurements, the methodology proposed by [19] has been implemented. This method assumes that the Doppler noise takes the form of a
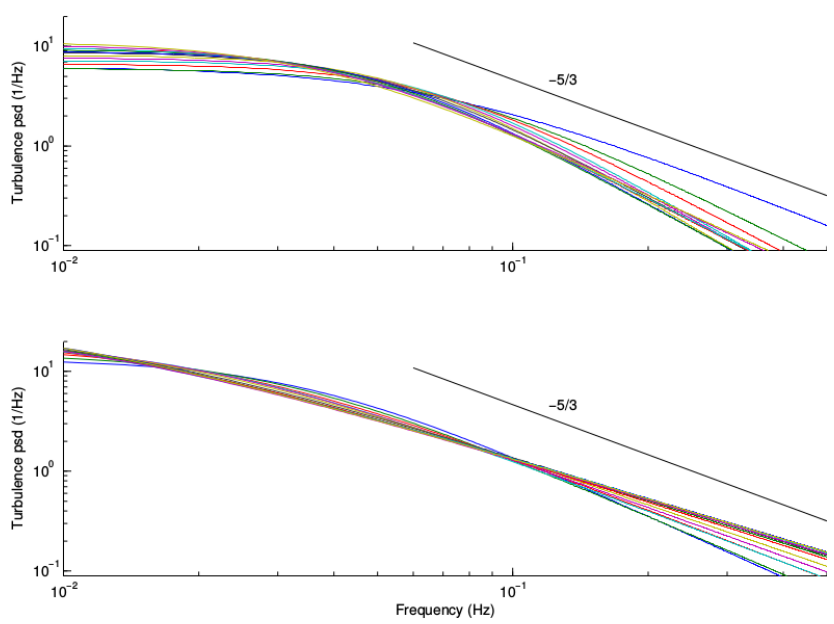

Fig. 14. Synthetic spectral densities from the analytical fit (see equation 3).

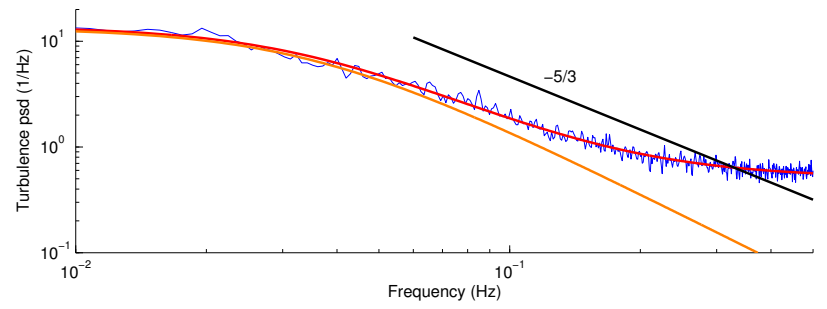

Fig. 15. Empirical spectral density (blue), fitted spectral model from equation 3 (red), the same with $\epsilon=0$ (no Doppler noise, orange).

white noise superimposed on the actual current signal. For one component of the current velocity this leads to:

$$
u(t)=u_{s}(t)+w_{n}(t)
$$

with $u(t)$ the measured velocity, $w_{n}(t)$ the white noise and $u_{s}(t)$ the actual velocity. Because $w_{n}(t)$ is not correlated to $u_{s}(t)$, the autocorrelation function of the actual velocity is:

$$
R_{u_{s}, u_{s}}(\tau)=R_{u, u}(\tau)-R_{w_{n}, w_{n}}(\tau) .
$$

As $R_{w_{n}, w_{n}}(\tau)=B$ (with $\mathrm{B}$ a constant) if $\tau=0$ and 0 otherwise, knowing $B$ yields the power spectral density of $u_{s}$ :

$$
S_{u_{s}, u_{s}}(f)=S_{u, u}(f)-S_{w_{n}, w_{n}}(f),
$$

By definition, $S_{w_{n}, w_{n}}(f)$, is a constant that we shall refer to as $S_{B}$. In practice, $S_{B}$ is estimated from the energy level in the high frequency region of $S_{u, u}(f)$, where the Doppler noise dominates the variance ([19]). For a clear illustration of the method the reader is invited to consult Figures 5 and 6 of [19]'s paper.

Figure 16 displays the result of this noise correction method applied to our dataset. It appears that the corrected spectrum (in red on the figure), loses the well-accepted $f^{-5 / 3}$ shape, characteristic of the turbulence inertial range. We believe the noise level estimated from the averaged energy level of $S_{u, u}(f)$ at frequencies above $0.4 \mathrm{~Hz}$ is overestimated. Indeed, the Nyquist frequency $\left(f_{N}=0.5 \mathrm{~Hz}\right)$ does not allow to observe the flattened portion of the spectrum representative of the Doppler noise variance. To obtain a more solid noise estimation, we assumed that the high frequency region of 


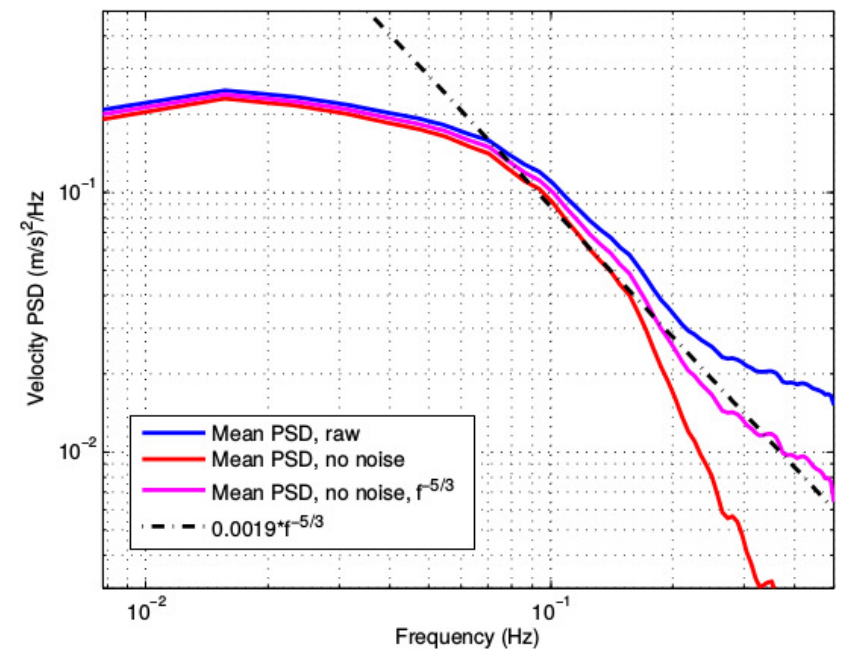

Fig. 16. Noise Correction illustration: blue initial spectrum, red noisecorrected spectrum, based on [19] and magenta red noise-corrected spectrum from the new approach presented here.

the spectrum should conform to the theoretical sub-inertial region $f^{-5 / 3}$ slope. We therefore developed an optimization algorithm, leading to the best estimation of the noise level based on the previous hypothesis. The slope is here assumed to start at frequencies not affected by the Doppler noise. Figure 16 reveals that the new corrected spectrum strongly differs: the spectral noise level given by our approach $\left(S_{B}=0.0088\right.$ $\left.(\mathrm{m} / \mathrm{s})^{2} / \mathrm{Hz}\right)$ is indeed roughly half that of the initial value. We are quite confident that our pragmatic method is reliable as the $f^{-5 / 3}$ slope has been reported in a number of studies dedicated to oceanic turbulence observation (e.g. [20],[21], [6]).

These results are in apparent disagreement with those of the previous section where a spectral flattening was clearly visible and extended towards lower frequencies (see Figures 5, 13). The difference comes from the contribution of the slack period $(\mathrm{U}>0.5 \mathrm{~m} / \mathrm{s})$ spectra that exhibit a clear flat section extending down to $0.3 \mathrm{~Hz}$ (Figure 17). These spectra were not considered in the present section as their signal-to-noise ratio are too high (e.g., [6], [19]). By construction, this can not be applied to the spectra produced by the stationarization method proposed earlier.

\section{B. Wave-turbulence separation: the coherence method}

We shall here investigate a 24-hour signal recorded on April 10, combining the effect of wave and turbulence. Due to the presence of waves, the stationarization method presented above can not be applied here.

1) Theoretical approach: Wave and turbulence often affect a similar spectral band. Therefore, the separation of both contributions is non-trivial and requires some assumptions on the wave and turbulence properties.

This time, we start from [4]'s approach but using the geographic velocities instead of the sum and difference beam velocities. The de-tided current velocity $u(t)$ is now assumed to be made of the turbulent and wave fluctuations $u_{t}(t)$ and $u_{w}(t)$, respectively, so that $: u(t)=u_{t}(t)+u_{w}(t)$.

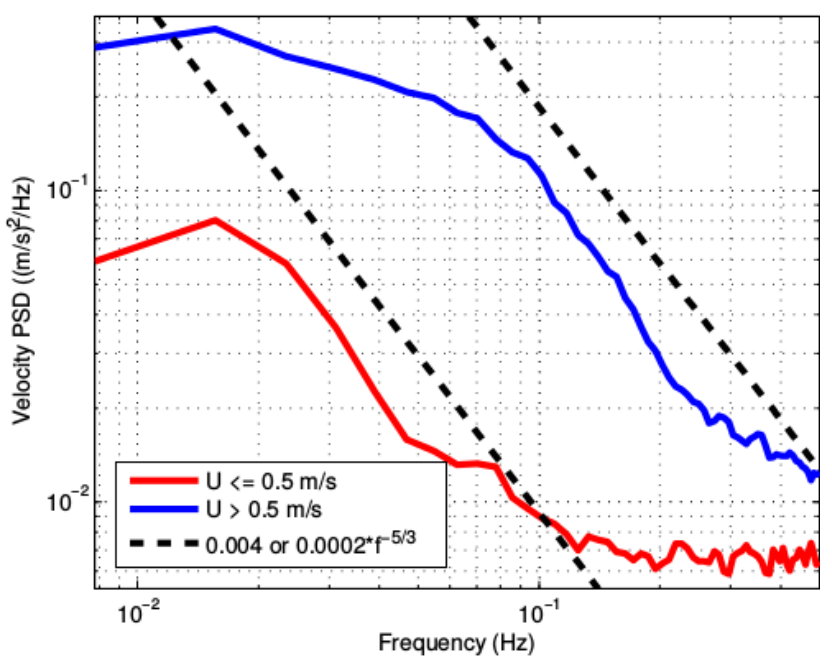

Fig. 17. Comparison of slack (red) and non-slack (blue) spectra (April 7), before Doppler noise correction.

The method then considers that the turbulent fluctuation are non-coherent over the water column:

$$
<u(t)>=<u_{w}(t)>
$$

Where $\langle.>$ stands for an average over the water depth $H$ : $\frac{1}{H} \int_{-H}^{0} \cdot d z$. The turbulence and noise fluctuation are further supposed to be statistically independent, yielding:

$$
S_{u}=S_{u_{w}}+S_{u_{t}}
$$

and for the cross-spectra:

$$
S_{u<u>}=S_{u_{w}<u>}
$$

These simplifying assumptions, combined with a few other straightforward calculations provide a very useful relation, linking the wave PSD and the total velocity PSD:

$$
S_{u_{w}}=\gamma_{u<u>}^{2} S_{u}
$$

Where $\gamma_{u<u>}$ is the squared coherence magnitude of the total velocity at a given altitude with the depth-averaged total velocity.

From the above relations and providing, the simplifying assumptions hold, it should be possible to separate the wave and turbulent contributions.

2) Idealized verification: The method was first tested in an idealized framework to verify that the implementation was correct. Wave orbital velocities were linearly generated from a Pierson-Moskowitz spectrum, characterizing a wave field with a significant wave height $H_{s}=4 \mathrm{~m}$ and peak period $T_{p}=12 \mathrm{~s}$ in $40 \mathrm{~m}$-depth. These conditions are representative of energetic wave conditions at our site. As for linear waves $\left\langle u_{w}(t)\right\rangle=$ $A \times u_{w}(t)$ with $A$ a function independent of the time, then the coherence $\gamma_{u_{w}<u_{w}>}^{2}$ should be unity. Our tests show that this is verified except for high non-dimensional depths $k D$ (with $k$ the wave number and $D$ the water depth) where the coherence is lost likely because the wave signal and the statistical noise commensurate (see figure 18). 


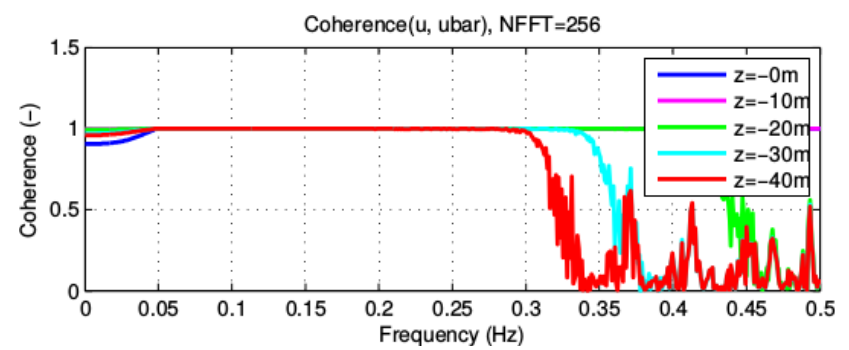

Fig. 18. Wave coherence test performed in the idealized case. Each colors correspond to a given depth.

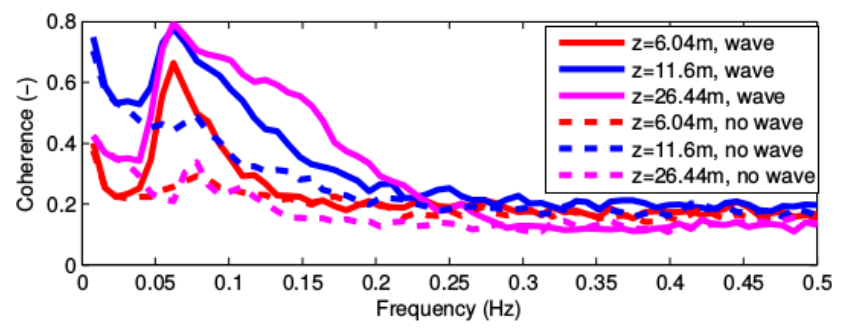

Fig. 19. Wave coherence at 6.04 and $26.44 \mathrm{~m}$ above seafloor. Continuous lines: April 10, dashed lines April 7.

3) Application to real data: To investigate the capability of the above method in separating the wave and turbulent fluctuations, a $24 \mathrm{~h}$-segment of the ADCP data was selected. This sample corresponds to April 10, 2013, a day characterized by high tidal velocities $\left(<u_{\max }>=2.3 \mathrm{~m} / \mathrm{s}\right)$ and a long period swell $\left(H_{s}=2 \mathrm{~m}, T_{p}=15 \mathrm{~s}\right)$.

The spectra of the de-tided, major axis velocity fluctuations are then estimated over $1024 \mathrm{~s}$ signal section sampled at $1 \mathrm{~Hz}$, filtered with 50\% overlapping Hanning windows and analyzed with 128 points Fast Fourier Transforms (fft), leading to 16 degrees of freedom.

The coherence $\gamma_{u_{w}<u_{w}>}^{2}$ is computed using the same segment length, number of fft points and overlapping Hanning windows. The $24 \mathrm{~h}$-averaged coherences at the lowest and highest altitudes available are displayed in Figure 19. For our application we choose to perform the analysis at bin 7 , whose altitude $(11.6 \mathrm{~m})$, approximately matches the hub-height of the tidal turbines tested at Paimpol-Bréhat.

Provided the underlying assumptions hold, the turbulent and wave spectra are distinguished, based on equations 8 and 10. To verify the accuracy of this approach we investigated how our turbulent spectra compare to turbulent spectra computed in wave-free conditions. For this, we picked the 24h-event on April 7, showing negligible wave orbital velocity at the target altitude (11.6 mab) and with similar tidal velocities. It is worth noting that this comparison assumes that the turbulence is not affected by waves.

For consistency, the spectra of both events were sorted in terms of the mean current magnitude and only those corresponding to a mean velocity falling between 0.5 and $1.5 \mathrm{~m} / \mathrm{s}$ were selected. The lower bound allows to remove slack period currents. Furthermore, an upper bound was needed, since the peak tidal velocities on April 10 were higher than on April 7. From Figure 20 it appears that the method fails in extracting the turbulent velocity spectra from the total velocity fluctuation

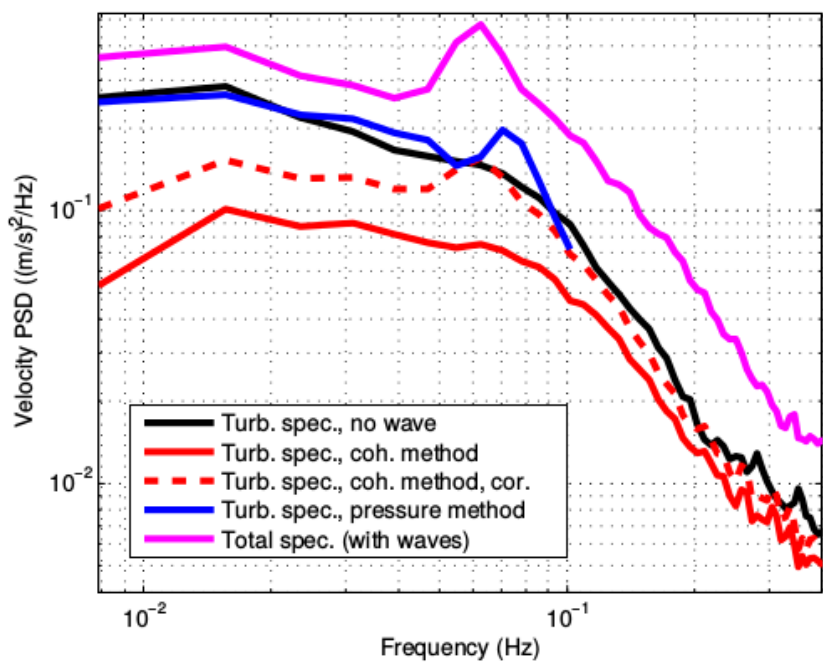

Fig. 20. Comparison of the wave-turbulence separation method. Black: average turbulent spectrum on April 7, no wave event, magenta: average spectrum on April 10 (wave event), red: turbulent spectrum on April 10, based on the coherence method, dashed red, same as red, but without the high frequency coherence noise contribution, blue: turbulent spectrum on April 10, from the pressure method. The displayed spectra have been noise corrected from the new method described in section IV-A.

spectra. The energy levels are largely underestimated meaning that the coherence is too high, all along the frequency axis.

The reasons for this are still not clear, but it seems that a spurious residual coherence remains at high frequency $(f>$ $0.3 \mathrm{~Hz}$ ). Correcting the coherence for that high frequency noise gives an excellent match to the reference spectrum at frequency above the spectral wave peak $(f>0.07 \mathrm{~Hz})$. However, the discrepancy is still evident at frequency $f<0.05 \mathrm{~Hz}$. This may suggest that the turbulent fluctuations are partly vertically coherent. This is indeed confirmed in figure 19, where the coherence grows significantly at frequencies less that $0.05 \mathrm{~Hz}$. Interestingly, the curves for the wave and wave-free events converge for $\mathrm{f}<0.05 \mathrm{~Hz}$, meaning that the coherence at these frequencies must be attributed to turbulent motions.

These preliminary conclusions demonstrate that the coherence is not a valid parameter to discriminate between wave and turbulent fluctuations for our field data. We further introduce an alternative method that uses the ADCP pressure measurements to infer the wave velocity spectrum.

\section{Wave-turbulence separation: the pressure method}

As shown in the previous section, the coherence method fails in separating the wave and turbulent motion because part of the turbulence is coherent over the vertical. The ADCP provides independent information on the wave characteristics through its pressure sensor and its surface tracking. The surface tracking data was too noisy to be used, we therefore focused our attention on the pressure data. From the linear theory, pressure spectra $S_{p}$ can be transformed into orbital velocity spectra $S_{u_{w}}$ through an appropriate transfer function:

$$
S_{u_{w}}=\left|\frac{2 \pi f}{\rho g} \frac{\cosh (k D)}{\sinh (k D)}\right|^{2} S_{p}
$$

with $\rho$ the water density, $g$ the gravity, $k$ the wave number and the $D$ the local water depth. The current has a strong effect 
on the wave field at our site, as an illustration, we found that a $2 \mathrm{~m} / \mathrm{s}$ current shifts the swell spectral peak from $0.06 \mathrm{~Hz}$ to $0.07 \mathrm{~Hz}$. This Doppler effect has been considered when computing equation 11. Interestingly, though the Doppler shift has a significant effect on individual spectra (computed every $1024 \mathrm{~s}$ ), it has negligible impact on the $24 \mathrm{~h}$-averaged spectrum. We believe this is due to a compensation of the flood and ebb-induced Doppler shifts. A cut-off frequency of $0.1 \mathrm{~Hz}$ has further been chosen to avoid noise amplification by the transfer function. Now recalling equation 8 the turbulent spectra $S_{u_{t}}$ can be deduced from:

$$
S_{u_{t}}=S_{u}-S_{u_{w}} \times|\cos (\theta)|^{2},
$$

where $\theta$ is the angle between the wave propagation direction and the current major axis. This has little effect in our situation as during the wave event (April 10), the wave direction deviated from less than $20^{\circ}$ from the current major axis. This method assumes linear waves and no directional spreading, but applying equation 12 provides a good agreement with the observations over the frequency band covered by the pressure-derived velocity spectra. The turbulent energy level is slightly overestimated around $0.08 \mathrm{~Hz}$ but our approach corrects for the low frequency underestimation reported with the coherence method. The method could be extended toward higher frequency ( $\mathrm{f}>0.1 \mathrm{~Hz}$ ) with good quality surface tracking data and/or in lower depths with pressure data. At the exception of the unexplained overestimation of the wavecorrected velocity PSD at $0.08 \mathrm{~Hz}$, the results suggest that the turbulence is not affected by the presence of waves at our site.

\section{CONCLUSIONS}

Through the present work, we have attempted to improve the knowledge of the wave and turbulent characteristics of a tidal energy site. For turbulent flow only, we have proposed a stationarization method that allows to obtain more rigorous and consistent estimations of velocity power spectral densities. The produced PSD exhibit quite similar shape regardless of the current axis (major and minor). The spectral shapes appear however to depend on the elevation in the water column. Based on these empirical findings an analytical fit is proposed and the synthetic turbulent spectra could be used as input to the numerical models run for production optimization and structural loading computations. In addition, the Doppler noise was shown to be dependent on the velocity variance whether it is related to wave or turbulent motion. We also found that waves introduce a higher Doppler noise level. An original approach is further proposed to correct the velocity spectrum from the Doppler noise, by forcing the spectral shape to conform to the widely accepted $f^{-5 / 3}$ slope in the frequency region affected by the noise. We also report that the turbulent motions are coherent over the vertical, at least for frequencies below $0.05 \mathrm{~Hz}$. This demonstrates that wave-turbulence separation methods based on the wave-coherence assumption are not valid, at least at our site. An alternative approach, relying on the estimation of the wave spectrum from the ADCP pressure data showed good ability in retrieving the turbulent spectrum in environment combining wave and turbulence. This indicates that the presence of waves may not affect the turbulence level. Finally, the velocity averaging of different cells at a given altitude induces a bias on the the orbital velocities estimation as soon as the wave length commensurates with the distance separating cells. It would then interesting to use the beam velocities, especially for turbulence analysis. These issues will be the topic of further research.

\section{ACKNOWLEDGMENT}

This work was part of the AESTUS project, co-funded by Alstom, DCNS, EDF, France Energies Marines, Ifremer, Ixsurvey and Open Ocean.

\section{REFERENCES}

[1] I. Milne, R. Sharma, R. Flay, and S. Bickerton, "The role of onset turbulence on tidal turbine blade loads," in Proc. 17th Australasian Fluid Mechanics Conf., Auckland, New Zealand, 5-9 December 2010, 2010.

[2] B. Gaurier, P. Davies, A. Deuff, and G. Germain, "Flume tank characterization of marine current turbine blade behaviour under current and wave loading," Renewable Energy, vol. 59, pp. 1-12, 2013.

[3] E. A. Terray, M. A. Donelan, Y. C. Agrawal, W. M. Drennan, K. K. Kahma, A. J. Williams, P. A. Hwang, and S. A. Kitaigorodskii, "Estimates of kinetic energy dissipation under breaking waves," J. Phys. Oceanogr., vol. 26, pp. 792-807, 1996.

[4] A. Hoitink, H. Peters, and M. Schroevers, "Field verification of adcp surface gravity wave elevation spectra," Journal of atmospheric and oceanic technology, vol. 24, no. 5, pp. 912-922, 2006.

[5] J. Thomson, B. Polagye, M. Richmond, and V. Durgesh, "Quantifying turbulence for tidal power applications," in OCEANS 2010. IEEE, 2010, pp. 1-8.

[6] J. Thomson, B. Polagye, V. Durgesh, and M. C. Richmond, "Measurements of turbulence at two tidal energy sites in puget sound, wa," Oceanic Engineering, IEEE Journal of, vol. 37, no. 3, pp. 363-374, 2012.

[7] K. Korotenko, A. Sentchev, and F. G. Schmitt, "Effect of variable winds on current structure and reynolds stresses in a tidal flow: analysis of experimental data in the eastern english channel," Ocean Science, vol. 8, no. 6, pp. 1025-1040, 2012.

[8] G. Voulgaris, D. Simmonds, D. Michel, H. Howa, M. B. Collins, and D. A. Huntley, "Measuring and modelling sediment transport on a macrotidal ridge and runnel beach: an intercomparison," Journal of Coastal Research, vol. 14, pp. 315-330, 1998.

[9] Y. Lu and R. G. Lueck, "Using a broadband adcp in a tidal channel. part ii: Turbulence," Journal of Atmospheric and Oceanic Technology, vol. 16, no. 11, pp. 1568-1579, 1999.

[10] P. J. Wiles, T. P. Rippeth, J. H. Simpson, and P. J. Hendricks, "A novel technique for measuring the rate of turbulent dissipation in the marine environment," Geophysical Research Letters, vol. 33, no. 21, 2006.

[11] A. C. Whipple, R. A. Luettich Jr, and H. E. Seim, "Measurements of reynolds stress in a wind-driven lagoonal estuary," Ocean Dynamics, vol. 56 , no. $3-4$, pp. 169-185, 2006.

[12] J. H. Rosman, J. L. Hench, J. R. Koseff, and S. G. Monismith, "Extracting reynolds stresses from acoustic doppler current profiler measurements in wave-dominated environments," Journal of Atmospheric and Oceanic Technology, vol. 25, no. 2, pp. 286-306, 2008.

[13] E. Boudière, C. Maisondieu, F. Ardhuin, M. Accensi, L. Pineau-Guillou, and J. Lepesqueur, "A suitable metocean hindcast database for the design of marine energy converters," International Journal of Marine Energy, vol. 3, pp. e40-e52, 2013.

[14] H. Tolman, "the wavewatch iii $($ ) development group. 2014. user manual

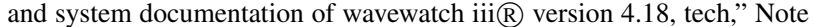
316, NOAA/NWS/NCEP/MMAB, 282 pp.+ Appendices, Tech. Rep.

[15] E. Droniou, "Contrôle qualité et analyse du courant moyen et de la turbulence à partir des données ADCP EM-SMADCP-bréhat-1," France Energies Marines, Tech. Rep., 2013.

[16] V. I. Nikora and D. G. Goring, "Adv measurements of turbulence: Can we improve their interpretation?" Journal of Hydraulic Engineering, vol. 124 , no. 6, pp. 630-634, 1998

[17] C. M. García, M. I. Cantero, Y. Niño, and M. H. García, "Turbulence measurements with acoustic doppler velocimeters," Journal of Hydraulic Engineering, vol. 131, no. 12, pp. 1062-1073, 2005. 
[18] J.-B. Richard, J. Thomson, B. Polagye, and J. Bard, "Method for identification of doppler noise levels in turbulent flow measurements dedicated to tidal energy," International Journal of Marine Energy, vol. 3, pp. 52-64, 2013.

[19] V. Durgesh, J. Thomson, M. C. Richmond, and B. L. Polagye, "Noise correction of turbulent spectra obtained from acoustic doppler velocimeters," Flow Measurement and Instrumentation, vol. 37, pp. 29-41, 2014.

[20] S. A. Kitaigorodskii, M. A. Donelan, J. L. Lumley, and E. A. Terray, "Wave-turbulence interactions in the upper ocean. part II: statistical characteristics of wave and turbulent components of the random velocity filed in the marine surface layer," J. Phys. Oceanogr., vol. 13, pp. 19881999, 1983.

[21] G. P. Gerbi, J. H. Trowbridge, E. A. Terray, A. J. Plueddemann, and T. Kukulka, "Observations of turbulence in the ocean surface boundary layer: Energetics and transport," Journal of Physical Oceanography, vol. 39, no. 5, pp. 1077-1096, 2009. 